\title{
Utilização de armadilhas para a captura de machos de Euglossini (Hymenoptera, Apoidea) ${ }^{1}$
}

\author{
Lácio A. O. Campos ${ }^{2}$ \\ Fernando A. da Silveira ${ }^{3}$ \\ Márcio L. de Oliveira ${ }^{4}$ \\ Célio V. M. Abrantes 2 \\ Elder F. Morato ${ }^{3}$ \\ Gabriel A. R. de Melo ${ }^{2}$
}

\begin{abstract}
The use of a commercial fruit fly trap, baited with atractive compounds, for the collection of male euglossine bees is described. Some of the preliminary results obtained are presented.
\end{abstract}

\section{INTRODUÇÃO}

As abelhas da tribo Euglossini são elementos típicos da fauna neotropical, ocorrendo da Argentina ao norte do México (PEARSON \& DRESSLER, 1985). Elas são abelhas ariscas, de vôo rápido e relativamente raras nas flores. Devido a isso, durante muitos anos, estiveram mal representadas nas coleções entomológicas.

A investigação das relaçöes entre machos de Euglossini e as flores de várias espécies de orquídeas levou a descoberta de uma série de compostos orgânicos altamente atrativos a essas abelhas (DODSON et al., 1969). A partir daí, vários estudos sobre a composição faunística sazonalidade e dispersão das abelhas Euglossini foram realizadas em várias localidades, com a utilização de iscas aromáticas (RICKLEFS et al., 1969; BRAGA, 1976; JANZEN et al. 1982; ACKERMAN, 1983; PEARSON \& DRESSLER, 1985; HARRIETT-POWELL, 1987; RAW, 1989).

Com a utilização desses compostos atrativos, grande número de exemplares foram coletados, principalmente de machos, que até entâo eram raríssimos nas coleções (MOURE, 1978). Isto possibilitou um completo reestudo da taxonomia de Euglossini (DRESSLER, 1978a,b, 1982a,b,c,; KIMSEY, 1977, 1982). Essa tribo teve o número de espécies descritas elevadas em cerca de 50\% entre 1969 e 1986 (KIMSEY \& DRESSLER, 1986).

$\mathrm{Na}$ maioria das coletas já realizadas, as abelhas eram capturadas com redes entomológicas ao serem atraídas pelas substâncias aromáticas (em geral expostas em papel filtro ou mata-borrão). Este procedimento, exige que o coletor permaneça junto às "iscas" durante todo o período de coleta.

1. Os dados coletados em Paraopeba são parte da tese de mestrado do segundo autor.

2. Dept. Biol.Geral, U.F.V. 36570 Viçosa-MG

3. Dept. Biol. Animal, U.F.V. 36570 Viçosa.MG

4. PDBFF, WWF/INPA. Caixa Postal, 478, 69011 Manaus.AM 
A primeira referEncia à captura de machos de Euglossini em armadilhas foi feita por LOPEZ (1963). Duzentos e vinte machos e 2 fêmeas de Eulaema tropica (Eulaema polychroma, segundo BENNETT, 1972) foram coletadas em armadilhas modelos Mc Phail e Steiner "carregadas" $\operatorname{com} \alpha-\mathrm{e} \beta$-ionona. Estas substâncias estavam sendo testadas para a captura de moscas-das-frutas, no México.

BENNETT (1972), utilizando armadilhas modelo McPhail, em Belize e FOLSOM (1985), utilizando armadilhas por ele mesmo desenvolvidas, na Colômbia, obtiveram sucesso na coleta de Euglossini, com o emprego de substâncias aromáticas como "iscas".

Aqui, é proposta a adaptação, para a coleta de machos de Euglossini, de uma armadilha comercializada no Brasil para a captura de moscas-das-frutas. Os procedimentos para a utilização dessa armadilha, bem como resultados iniciais obtidos, são discutidos.

\section{MATERIAL E MÉTODOS}

A armadilha utilizada foi o modelo "biologico" da Melpan Produtos Agrícolas Ltda. (Santo Antônio de Posse, São Paulo). Ela consiste de um tronco de cone oco, confeccionado em material plástico rígido e translúcido e contém lateralmente quatro orifícios afunilados. $\mathrm{O}$ fundo, atarrachado ao corpo da armadilha, possui também um orifício afunilado central. $O$ conjunto é fechado por um tampa rosqueada (Fig. 1).

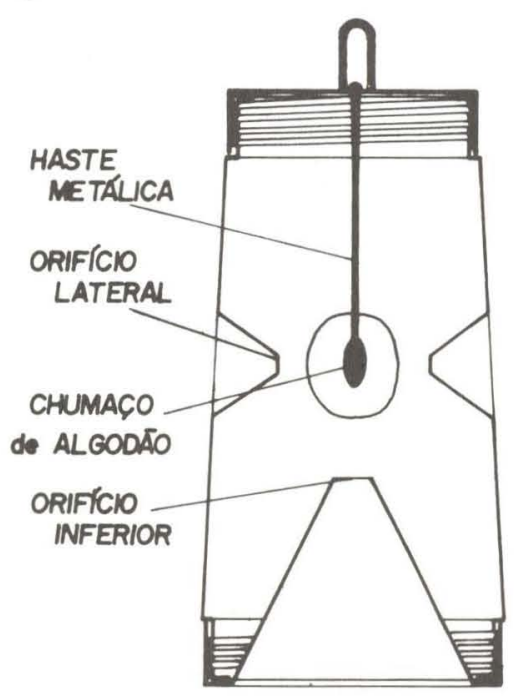

As adaptaçãoes realizadas para a captura das Euglossini foram as seguintes: aumento do diâmetro dos orifícios laterais, para cerca de 13 $\mathrm{mm}$ e fixação de uma haste metálica de aproximadamente $10 \mathrm{~cm}$ de comprimento sob a tampa. $\mathrm{Na}$ extremidade desta haste, à altura dos orificios laterais, ajusta-se um chumaço de algodaó, que é embebido na substância atrativa.

A armadilha assim preparada é dependurada em local aberto, preferencialmente ao abrigo da insolação direta. Nos testes realizados, até o momento, as armadilhas tem sido instaladas a uma altura de cerca de $1,80 \mathrm{~m}$ do solo.

Fig. 1. Esquema da Armadilha.

Foram realizadas, até agora, coletas em matas semi-decíduas secundárias, em Viçosa, Juiz de Fora e Ponte Nova (MG), no cerrado secundário em Paraopeba (MG), em mata seca e no cerrado em Minas Novas (MG), em campo rupestre em Diamantina (MG), em mata pluvial e savana na ilha de Maracá (RR) e em floresta pluvial em Manaus e em Tefé (AM). Diferentes compostos foram utilizados em cada 
uma dessas áreas, de acordo com a disponibilidade de armadilhas e substâncias atrativas (CAMPOS e col., dados não publicados).

Em Paraopeba, paralelamente a um levantamento das abelhas em visita às flores em vegetação de cerrado (SILVEIRA, 1989), foi tentado o primeiro levantamento periódico de Euglossini com a utilização das armadilhas. Neste estudo, empregaram-se apenas duas armadilhas de cada vez, cada qual com um dos seguintes compostos: óleo de cravo, acetato de benzila e salicilato de metila. As armadilhas foram instaladas mensalmente, em geral durante dois dias consecutivos, sempre no mesmo local, por um período de mais ou menos nove horas (de cerca de 7:30 h às 16:30 h). Ao todo foram 15 dias de coletas, em sete meses.

Em Manaus, foram utilizados cineol, óleo de cravo, salicilato de metila e acetato de benzila. Foram realizadas amostragens, no interior e borda da floresta pluvial, bem como em uma área desmatada. Três jogos de armadilhas eram instalados, concomitantemente, em um ponto amostral, em cada ambiente. Em dias consecutivos, outros dois pontos eram amostrados, em cada um daqueles ambientes. Este procedimento era repetido a cada 15 dias e as armadilhas permaneciam expostas durante 24 horas. Cada ponto foi amostrado 9 vezes, entre agosto de 1988 e janeiro de 1989. (CAMPOS \& MORATO, não publicado).

Em Viçosa, seis conjuntos de cinco armadilhas (acetato de benzila, eugenol, salicilato de metila, vanilina e cineol) foram utilizados ao mesmo tempo, um no interior e um na borda de três manchas de mata. As armadilhas permaneciam instaladas por 24 horas consecutivas, em intervalos de 15 dias, ao longo de 16 meses de amostragem (CAMPOS \& ABRANTES, não publicado).

Em Ponte Nova, onde ainda estão sendo realizadas coletas quinzenais, têm-se utilizado cineol, cinamato de metila, eugenol, vanilina, escatol, acetato de benzila e salicilato de metila, em dois pontos à margem de mata secundária.

\section{RESULTADOS}

Em torno de 40 espécies diferentes foram capturadas, atraídas pelas diversas "iscas" aromáticas, nos diversos locais amostrados. Com os orifícios de $13 \mathrm{~mm}$ de diâmetro foram coletados desde Eulaema meriana, (largura máxima do tórax medida em espécimes secos alfinetados $=14 \mathrm{~mm}$ ) até Euglossa $\mathrm{sp}$. (3.2 mm de largura máxima do tórax).

$\mathrm{O}$ número de indivíduos capturados em cada dia de coleta variou de local para local e de "isca" para "isca". Em Paraopeba, a única substância que se mostrou atrativa, entre as utilizadas, foi o bleo de cravo, cujo principal componente é o eugenol. Aí, o número máximo de indivíduos coletados, numa única armadilha, num único dia de amostragem, foi de 10 abelhas (nove de uma espécie e uma de outra). Um total de 42 indivíduos pertencentes a três espécies de Euglossa foram capturados nos 15 dias de coleta entre, março e outubro de 1987.

Em Manaus, a amostragem realizada, durante 6 meses permitiu a coleta de 838 indivíduos de várias espécies de Euglossa, Eulaema, Euplusia, Eufriesea e Exaerete. Nesse período, o número máximo de indivíduos coletados, numa única armadilha, em um dia de coleta, foi 62 (CAMPOS \& MORATO, não publicado).

Em Viçosa, 1053 indivíduos de várias espécies de Euglossa, Eulaema, Euplusia, e Exaerete foram coletados nos 33 dias de coleta, ao longo de 16 meses. O nú- 
mero máximo de indivíduos coletados em uma única armadilha, num único dia de coleta, foi 23 (CAMPOS \& ABRANTES, não publicado).

Em Ponte Nova, têm sido coletadas Eulaema, Euglossa e Exaerete. Aí, o número máximo de abelhas coletadas em uma única armadilha, até o momento, foi 63 (CAMPOS \& SILVEIRA, não publicado).

$\mathrm{O}$ número de indivíduos coletados pelas armadilhas também variou em dias consecutivos. A armadilha instalada com óleo de cravo, em Paraopeba, coletou uma média de 2,8 espécimes por dia. Em maio de 1987, entretanto, ela coletou respectivamente 1 e 10 abelhas em cada um dos dois dias consecutivos de amostragem. $O$ número mínimo de abelhas coletadas em um único mês (dois dias de amostragem), em Paraopeba, foi 3.

Em Paraopeba, três espécies, Euglossa melanotricha, E, modestior e E. sp. foram capturadas em armadilha, atraídas por bleo de cravo e eugenol na área de cerrado. Uma fêmea de Euglossa modestior foi capturada enquanto voava insistentemente em torno da rede entomológica, a cerca de $400 \mathrm{~m}$ das armadilhas. Nenhuma dessas espécies, entretanto, foi encontrada em visita às flores. E. townsendi, por outro lado, apesar de encontrada freqüentemente nas flores (machos e fêmeas), jamais foi capturada pelas armadilhas. Em função de sua timidez e velocidade de vôo, muitos indivíduos de Euglossa escaparam às tentativas de captura com a rede entomológica, enquanto visitavam flores em Paraopeba.

Várias outras espécies de abelhas (por exemplo, Lestrimelitta sp., Ptiloglossa spp. e Megalopta spp), de outros insetos (coleopteros, neuropteros, diptera etc.), e aranhas têm sido capturados com freqüência nas armadilhas.

\section{DISCUSSÃO}

JANZEN et al. (1982) crêem que as Euglossini, dada a sua grande capacidade de vôo, percorram distâncias de algumas dezenas de quilômetros na execução de suas tarefas. Dessa forma, diferentes atividades poderiam ser desempenhadas em diferentes sub-habitats e machos e fêmeas, portanto, poderiam não estar presentes nos mesmos locais, enquanto realizassem diferentes tarefas. Talvez essa seja a razão pela qual, em Paraopeba, as fêmeas das espécies de Euglossini, cujos machos foram capturados nas armadilhas, nunca tenham sido capturados nas flores.

A ausência, nas armadilhas, em Paraopeba de Euglossa townsendi, aí comumento coletada nas flores, é também curiosa. $\mathrm{Na}$ Costa Rica, esta espécie foi atraída por eugenol (JANZEN et al., 1982), o principal componente do бleo de cravo. Por outro lado, existem evidências de que a mesma substância exerça atratividades diferentes, para a mesma espécie, em diferentes locais (JANZEN et al., 1982). É possível, portanto, que aquela espécie tivesse sido atraída se outros compostos houvessem sido empregados como "isca".

As armadilhas se mostraram bastante eficientes na coleta de Euglossini. Espécies de todos os gêneros da tribo (exceção feita a Aglae, bastante raro), incluindo toda a gama de tamanhos conhecidos para essas abelhas, foram capturados por elas.

Em Paraopeba, ficou evidente que a restrição da amostragem às abelhas encontradas nas flores, seria insuficiente para detectar as espécies capturadas em armadilhas. Elas, portanto, se mostraram um método apropriado para o levantamento 
da fauna de Euglossini, paralelamente a outros métodos de amostragem para outros Apoidea, uma vez que não exigem a presença do coletor.

\section{AGRADECIMENTO}

Os autores expressam seu agradecimento ao Prof. Pe. Moure, da Universidade Federal do Paraná, pela identificação das Euglossa capturadas em Paraopeba, a Eduardo Hickel, pela confecção do desenho da armadilha, na Fig. 1, e ao CNPq, pelos auxílios concedidos.

\section{REFERÊNCIAS}

ACKERMAN, J.D. 1983. Diversity and seasonality of male euglossine bees (Hymenoptera: Apidae) in Central Panama. Ecology, 64 (2):274-283.

BENNETT, F.D. 1972. Baited McPhail fruitfly traps to collect euglossine bees. New York Entomological Society, 80: 137-145.

BRAGA, P.I.S. 1976. Atração de abelhas polinizadoras de Orchidaceae com o auxílio de iscasodores na campina, campinarana e floresta tropical úmida da região de Manaus. Ciência e Cultura, 28 (7):767-773.

DODSON, C.H.; DRESSLER, R.L.; HILLS, H.G.; ADAMS, R.M.; WILLIAMS, N.H. 1969. Biologically active compounds in orchid fragrances. Science, 164: 1243-1249.

DRESSLER, R.L. 1978a. New species of Euglossa from Mexico and Central America. Revista de Biologia Tropical, 26 (1): 167-185.

DRESSLER, R.L. 1978b. An infrageneric classification of Euglossa, with notes on some features of special taxonomic importance (Hymenoptera: Apidae). Revista de Biologia Tropical, 26 (1): 187-198.

DRESSLER, R.L. 1982a. New species of Euglossa. II. (Hymenoptera: Apidae). Revista de Biologia Tropical, 30 (2):121-129.

DRESSLER, R.L. 1982b. New species of Euglossa. III. The bursigera species group (Hymenoptera:Apidae). Revista de Biologia Tropical, 30(2):131-140.

DRESSLER, R.L. 1982c. New species of Euglossa. IV. The cordata and purpurea species groups (Hymenoptera: Apidae). Revista de Biologia Tropical, 30(2):141-150.

FOLSOM, J.P. 1985. Dos nuevas tecnicas para capturar y marcar abejas machos de la tribu Euglossini (Hymenoptera: A pidae). Actualidades Biologicas, 14(51):20-25.

HARRIETT POWELL, A.\& POWELL, G.V.N. 1987. Population dynamics of male Euglossine bees in amazonian forest fragments. Biotropica, 19(2):176-179.

JANZEN, D.H.; DEVRIES, P.J.; HEGGINS, M.L.; KIMSEY, L.S. 1982. Seasonal and site variation in Costa Rican euglossine bees at chemical baits in lowland deciduous and evergreen forests. Ecology, 63(1):66-74.

KIMSEY, L.S. 1977. New species of bees in the genera Euplusia and Eufriesea (Hymenoptera: Apidae: Euglossini). The Pan-Pacific Entomologist, 53: 8-18.

KIMSEY, L.S. 1982. Systematics of bees of the genus Eufriesia (Hymenoptera: Apidae). University of California Publications in Entomology, 95: 1-125.

KIMSEY, L.S. \& DRESSLER, R.L. 1986. Synonymic species list of Euglossini. Pan-Pacific Entomologist 62(3): 229-236. 
LOPEZ D., F. 1963. Two attractants for Eulaema tropica L. Journal of Economic Entomo$\operatorname{logy}, 56(4): 540$.

MOURE, J.S. 1978. Reestudo dos tipos de algumas espécies de Euplusia descritos por Friese (Hymenoptera: Apidae. Studia Entomologica, 20(1-4): 253-267.

PEARSON, D.L. \& DRESSLER, R.L. 1985. Two-year study of male orchid bee (Hymenoptera: Apidae: Euglossini) Attraction to chemical baits in lowland south-eastern Peru. Journal of Tropical Ecology 1:37-54.

RAW, A 1989. The dispersal of Euglossini bees between isolated patches of eastern Brazilian wet forest (Hymenoptera, Apoidea). Revista Brasileira de Entomologia, 33(1): 103-107.

RICKLEFS, R.E.; ADAMS, R.M.; DRESSLER, R.L. 1969. Species diversity of Euglossa in Panama. Ecology 50(4): 713-716.

SILVEIRA, F.A. DA 1989. Abelhas Silvestres (Hymenoptera: Apoidea) e suas Fontes de Aleimento no Cerrado da Estação Florestal de Experimentação de Paraopeba, Minas Gerais Viçosa, Universidade Federal de Viçosa. 50 p. (Tese M.S.). 